\title{
Repetitive Transcranial Magnetic Stimulation as a Probe of Episodic Memory Neurocircuitry in Schizophrenia
}

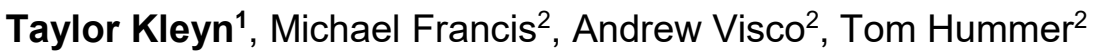

${ }^{1}$ Indiana University School of Medicine; ${ }^{2}$ Department of Psychiatry, Indiana University School of Medicine

\section{Background and Hypothesis:}

People with schizophrenia often experience impairments with episodic memory (EM). Due in part to a lack of understanding regarding the neural mechanisms of EM, there are no effective treatments. Recent research indicates that the precuneus may be associated with EM impairment. Repetitive transcranial magnetic stimulation (rTMS) is a commonly employed intervention for treatment resistant depression, but its potential for investigating other psychiatric disorders such as schizophrenia is unclear. We hypothesize that, compared to sham stimulation, $1 \mathrm{~Hz}$ rTMS will decrease precuneus activity and $20 \mathrm{~Hz}$ rTMS will increase precuneus activity during an EM task.

\section{Experimental Design:}

Seven patients with early phase psychosis underwent a baseline fMRI scan during an EM recognition task that required participants to accurately identify which images were previously shown (targets) or not shown (foils). Next, participants had three separate rTMS sessions targeting the precuneus, each one week apart, in a randomized order: inhibitory $(1 \mathrm{~Hz}) \mathrm{rTMS}$, excitatory $(2 \mathrm{~Hz}) \mathrm{rTMS}$, and sham stimulation. Each rTMS session was immediately followed by fMRI during the EM task.

\section{Results:}

We currently remain blind to the conditions because the study is ongoing. Participants had relatively lower accuracy during foil trials in one treatment session. During this same session, precuneus activity was relatively stronger to foils than targets, compared to other treatment sessions. These preliminary results suggest that rTMS applied to the precuneus may impact episodic memory and related brain activity in early psychosis

\section{Conclusion and Potential Impact:}

Data from this study will help determine whether targeting the precuneus with rTMS impacts functional activation in patients with schizophrenia during EM tasks. Additionally, relationships between EM performance and changes in precuneus activity will be identified. If effective, rTMS may represent a novel treatment for EM deficits in schizophrenia. 